REVIEW ARTICLE

Title

\title{
The Role of Inflammation in Chronic Kidney Disease
}

Authors:
Mochammad Thaha ${ }^{1}$, I Gde Raka Widiana ${ }^{2}$
1. Division of Nephrology, Department of Internal Medicine, Faculty of Medicine
Universitas Airlangga - Universitas Airlangga Hospital
2. Division of Nephrology, Department of Internal Medicine, Faculty of Medicine
Universitas Udayana - Sanglah General Hospital
Editor
Suhardjono

Received 8 April 2019, revised 22 October 2019, accepted 1 November 2019, published 2 December 2019

\begin{abstract}
The morbidity and mortality rate of patients with chronic kidney disease $(\mathrm{CKD})$ remains high despite progressive development in CKD management. Micro inflammation is an important component of CKD and has an important role in the pathophysiology of cardiovascular disease (CVD) complications, as well as a protein-energy deficiency (PED) and mortality. Various factors contribute to the inflammatory state of CKD, including increased production and decreased excretion of pro-inflammatory cytokines, oxidative stress, acidosis, chronic vascular access infection, fat metabolism dysregulation, and intestinal dysbiosis. The inflammatory process is directly correlated with glomerular filtration rate (GFR) in CKD and reaches its peak in end-stage renal disease (ESRD). Several dialysis-related factors such as bioincompatible membranes and dialysate quality also have important roles in the inflammatory process. Meanwhile, the role of genetic and epigenetic factors is being widely studied. Efforts in reducing inflammation such as lifestyle modification, medication, and dialysis optimization have been performed and tested in controlled clinical trials.
\end{abstract}

Keywords: inflammation, chronic kidney disease, uremic toxin

Corresponding author: Mochammad Thaha; e-mail:mochthaha@yahoo.com

\section{Introduction}

Inflammation as part of $\mathrm{CKD}$ has been linked with CVD, PED, and mortality. ${ }^{1,2}$ The interest in inflammation in CKD and ESRD has developed over many years, marked by the shift of inflammation from a novel subject to an established term. In this article, we review the latest development of uremic inflammation and its implications, the role of mediators of inflammation in hemodynamics, salt, and water homeostasis, as well as blood pressure. Uncontrolled inflammation leads to glomerular, tubular, and interstitial damage. Pathological disorders of the kidneys are associated with acute kidney disorders and chronic kidney disease. Inflammation is associated with cardiovascular disease and diabetes, which leads to organ damage including the kidney. ${ }^{3}$

\section{Inflammation and CKD Outcome}

It has been found that there's an inverse correlation between GFR and inflammation. In the Chronic Renal Insulin Cohort (CRIC) study, inflammatory markers (IL1B, IL-1 antagonist receptor, IL-6, TNF-L, CRP, and fibrinogen) were inversely associated with renal function and were directly proportional to albuminuria. ${ }^{4}$ Various inflammatory markers have different predictive values for CKD and ESRD. A study shows IL-6, WBC, and TNF could predict all-cause mortality risk. ${ }^{1}$

Specific roles of multiple cytokines are being investigated in CKD/ESRD, such as increased IL-2 in chronic 
HD patients with uremic pruritus compared to patients with non-uremic pruritus. ${ }^{5}$ Obesity-related CKD is associated with spleen derived IL-10. ${ }^{6}$ TNF-like weak inducer of apoptosis (TWEAK) is capable of stimulating the expression of inflammatory cytokines in renal cells and the down-regulation of in vitro and in-vivo excretion of klotho. ${ }^{7}$ Soluble TWEAK is associated with the severity of coronary heart disease (CHD) in stage 2-3 CKD. ${ }^{8}$

A study by Akchurin et al. in patients with progressive dependence towards HD reported high levels of pro-inflammatory cytokines IL-1, IL-6, and TNFL and low levels of anti-inflammatory cytokines IL-2, IL-4. IL-5, IL-12, CH 50 and T-Cell. This study showed that CKD patients with high levels of pro-inflammatory cytokines had a lower survival rate. ${ }^{6}$

Recent data show the importance of adipokine roles in CKD such as leptin, apelin, omentin, visfatin, resistin, and anti-inflammatory adipokine. ${ }^{9}$ A study by Lim et al. showed that CKD patients had a positive association with serum adiponectin and leptin level. ${ }^{10}$ Similarly, adiponectin levels are significantly higher in CKD patients than those with normal kidney function. ${ }^{11}$ At CKD stage 2-5, leptin levels are associated with metabolic syndrome scores and PTH levels. ${ }^{12}$

Persistent inflammation in CKD is not only related to CVD outcomes including atherosclerosis but also plays an important role in the onset of PED. Hypoalbuminemia is strongly associated with mortality in dialysis patients. The underlying condition is inflammation and not just malnutrition. ${ }^{13,14}$ There are several mechanisms suspected to cause PED. ${ }^{15}$ Proinflammatory cytokines directly affect the brain causing anorexia. In addition, inflammatory markers, particularly IL-6, are associated with the onset of depression in both CKD and ESRD and have predictive value for morbidity, morbidity and decreased nutritional intake. ${ }^{16}$ Inflammation not only causes malnutrition but also increases resting energy expenditure (REE). The immune system occupies the 3rd position in consuming body energy after brain and muscle. Furthermore, the inflammatory process inhibits anabolic hormones such as growth hormone $(\mathrm{GH})$, IGF-1 and testosterone. ${ }^{17}$

The consequences of inflammatory processes in renal disease also include anemia and mineral and bone metabolism disorders (CKD-MBD), including growth disorders in children with CKD. TNF-L as a potent cytokine in activating osteoclast and bone resorption can cause pathological fractures in patients with CKD. ${ }^{18}$ Munoz et al study reported high levels of FGF23 are independently associated with high levels of IL-6, CRP, TNF-L and fibrinogen (77). ${ }^{19}$ Meanwhile, IL-1 and IL-6 inhibit PTH secretion. Low levels of PTH illustrate more of the condition of malnutrition-inflammatory-cachexia syndrome instead of low bone turnover disease (33). ${ }^{20}$

Inflammation in CKD also plays an important role in the occurrence of anemia and erythropoietin resistance (Epo). ${ }^{21}$ Inflammatory effects on Epo are multifactorial, such as the inhibition in the production of Epo, suppression of the effects of EPO stimulation on erythropoiesis process, increased the production of hepcidin, and metabolic iron disorders. ${ }^{22}$

Another component that also contributes to the inflammatory process is oxidative stress. Oxidants are physiologically generated from tissue repair processes, for example after the invasion of microorganisms or other foreign antigens. However, a prolonged time of this process will have a detrimental effect and contribute to cell and tissue damage. ${ }^{23}$ Inflammation in patients with CKD can be triggered by activation of the complement system. Hemodialysis with cellulose and hemicellulose contributes to oxidant production and complement activation, resulting in worsening oxidative stress. The pathophysiology of inflammation differs between race, ethnicity, and genetic backgrounds. Crews et al reported in a cohort study the absence of survival rate in ESRD patients with low inflammation levels, whereas a racial-paradox was observed with the presence of a significantly higher survival rate in patients with high inflammation levels. ${ }^{24}$ Some studies reported the cardioprotective traits of certain IL-10 genotypes against CVD complications. ${ }^{25}$ Low levels of IL-10 can reduce symptoms in patients with ESRD.

\section{Etiology of inflammation in CKD}

Multiple factors influence immune system dysregulation and activation of inflammation in CKD; due to the underlying disease leading to CKD or the uremia caused by CKD itself. Other factors include lifestyle, diet, and environment depicting epigenetic impact. The high levels of proinflammatory cytokines can be the result of increased production and decreased excretion. ${ }^{6}$ Uremia increases the production of oxidative stress, carbonyl stress, and is further aggravated by iron supplementation in CKD patients. ${ }^{26}$ Metabolic acidosis is also an important cause of inflammation. ${ }^{27}$

CKD patients, especially with ESRD, tend to develop infection and thrombosis that can easily induce inflammation such as vascular access infection, intravenous fistula, and graft thrombosis, peritonitis episodes in peritoneal dialysis patients, and chronic periodontal inflammation, that can lead to the decline of survival rate. $^{3}$ 
Uremic toxins are also suspected to contribute to the presence of intestinal dysbiosis in CKD, leading to increased risk of bacterial translocation and systemically induce inflammation. ${ }^{28} \mathrm{~A}$ latest cross-sectional study in stage 3-4 CKD patients showed that indoxyl sulfate dan $\mathrm{p}$ cresyl sulfate (a reno-cardiac toxin produced by intestinal microbiota) is associated with increased inflammation biomarkers and CKD patients' arterial stiffness. $^{29}$

Vitamin D is widely known to have a role in the regulation of the immune system. Other than the inability to produce $1.25(\mathrm{OH}) 2$ vitamin $\mathrm{D}$, CKD patients also lose the ability to maintain an adequate concentration of $25(\mathrm{OH})$ vitamin $\mathrm{D}$. The deficiency of vitamin $\mathrm{D}$ and immune system dysfunction is thought to be a contributing factor to inflammation. ${ }^{30}$

In CKD patients, pro-inflammatory cytokines are also produced by other tissues such as adipose tissues and not just lymphocytes. Visceral adipose tissue of ESRD patients expresses pro-inflammatory mRNA such as TNF-L, CD68, adiponectin receptor-1, MCP-1. In stage 3-5 CKD, the visceral fat volume is correlated with IL$6 .{ }^{31}$ The effect of high levels of adiponectin receptor-1 in CKD might be suppressed by adiponectin resistance in the post-receptor level. ${ }^{32}$

Hemodialysis itself has been known to increase the transcription process of pro-inflammatory cytokines. Extracorporeal factors that induce inflammation include contaminated dialysate fluid, microbiological quality of dialysate fluid, and incompatible dialysis membranes.

\section{Therapy of Inflammation in CKD}

Therapy of the inflammatory process in CKD includes lifestyle modification, pharmacologic management, and dialysis optimization. Certain diets, oral vitamin supplementation, and physical exercise at home and during hemodialysis appear to be beneficial and economical for the reduction of mortality and improvement of quality of life in ESRD patients. ${ }^{33}$ The response to such treatments depends on the level of inflammation in CKD. ${ }^{34}$

Other than total nutritional intake, certain diet components affect the inflammatory process in CKD such as omega-3 fatty acid (fish oil), catechin, decaffeinated green tea, pomegranate juice, soy milk, fiber, and probiotics. ${ }^{35}$

The pharmacological intervention aims to suppress the inflammatory process as shown in experimental animal studies. Non-conventional anti-inflammatory intervention includes growth-hormone, vitamin D, new anti-inflammatory drugs, antioxidants, and specific anti-cyto6 kine therapy.

Traditional CKD treatments have been extensively studied. A meta-analysis showed statins can alleviate chronic inflammatory states in ESRD patients. ${ }^{36}$ angiotensin-receptor blocker olmesartan administration in CKD patient stage 3-4 for 16 weeks induced significant reduction in CRP and fibrinogen, but not adipokines. ${ }^{37}$

Oxidative stress from hemodialysis can be reduced through multiple interventions to dampen inflammatory cell activity and to decrease inflammation mediators. Dampening inflammatory cell activity is done using biocompatible synthetic dialysis membranes such as polysulfone and highly purified dialysate. The extraction of inflammatory mediators can be done through hemolipodialysis, using a dialysis membrane coated with vitamin E and electrolyzed reduced water. Treatments of oxidative stress have developed extensively, such as gamma-tocopherol, docosahexaenoic acid, $\mathrm{N}$-acetylcysteine, bardoxolone methyl and vitamin E-coated dialyzer membrane. ${ }^{38}$ In a randomized controlled study, gamma-tocopherol and docosahexaenoic significantly reduce IL-6, leukocyte, and neutrophil in dialysis patients, although they don't reduce CRP, F2 isoprostane, and protein carbonyl. ${ }^{39}$ A meta-analysis showed vitamin E-coated dialyzer membrane significantly reduced inflammation and oxidative stress without affecting the adequacy of the dialysis itself. ${ }^{40}$

Cytokine targeted therapy has also shown promising results and research has been done in patients without CKD as well. Specific anti-IL-1, Il-6, and TNF therapy in CKD patients are still limited. Administration of IL-1 receptor antagonist in dialysis patients has been shown to reduce CRP and IL-6, and increased albumin, prealbumin, lean body mass, and adiponectin. ${ }^{41}$ For dialysis patients, ideal therapy for reducing inflammation includes eliminating inflammatory factors and mediators, using biocompatible dialyzer membranes, good quality water, ultrapure dialysate, increased dialysis frequency, and hemodiafiltration.

The latest meta-analysis showed the use of ultrapure dialysis reduced inflammatory markers and oxidative stress, and increased albumin and hemoglobin, reducing the need for EPO. Frequent and short dialysis is more favorable in reducing inflammation and increasing EPO sensitivity, while extended/frequent dialysis promotes better outcomes for CVD. ${ }^{42}$ Hemodiafiltration reduces inflammatory activity by increasing medium-sized molecules clearance by convection. A study by Ağbaş et al showed online hemodiafiltration could reduce inflammation marked by reduced IL-6 and CRP levels compared to low-flux hemodialysis. ${ }^{43}$ Mercadal et al study concluded that hemodiafiltration had better cumulative survival regardless of dialysis

InaKidney | Vol. II | Is. 3| Sept - Dec 2019 
prescription. ${ }^{44}$

In conclusion, persistent microinflammation is inseparable from CKD. It contributes to the development of various complications such as CVD, malnutrition, etc. and mortality. The factors contributing to this condition include reduced excretion of inflammatory cytokines, oxidative stress, acidosis, recurrent infection, adipose tissue, poor dialysate water quality, and incompatible dialyzer membrane. Interventional therapy includes lifestyle modification, pharmacological therapy, and dialysis optimization. Further study is much needed in the future.

Activation of the immune system is the most important factor in the progression of kidney disease both acute and chronic. The immune system is composed of innate and adaptive immunity. The innate immunity is the first line of defense when pathogens are first introduced to the body and is not specific, whereas the adaptive immunity serves as a specific immunity after the initial encounter of pathogens with innate immunity.

\section{Innate Immunity in CKD}

Toll-like receptors (TLR) have been linked to acute kidney injury (AKI) and CKD in several studies. TLR are proteins that act as an identifier of pathogenic proteins, which will bind pathogenic antigens and stimulate inflammatory responses. TLR correlates directly with $\mathrm{AKI}$ and is known to have a pathogenic role in $\mathrm{CKD} .{ }^{45}$

Dendritic cells also have an important role in inflammation, being the activator of T cells. Macrophages are also found in kidneys of CKD patients. Macrophages are activated after the activation of the complement system or $\mathrm{T}$ cells effector in the kidney, leading to the possibility of macrophage not being the primary factor in CKD progression. ${ }^{46}$ However, several studies found AKI, CKD, and immune-mediated glomerulonephritis are correlated with the number of macrophages found in the kidney. ${ }^{47}$ Natural killer cells are also known to cause macrophage activation, contributing to inflammation. ${ }^{48}$ Osteopontin is also a factor responsible for the recruitment of macrophages and can increase during proteinuria. ${ }^{49}$

\section{Adaptive immunity and CKD}

The adaptive immunity is mainly regulated by the activity of B and T cells. B lymphocytes produce an autoantibody that plays a role in kidney disease. ${ }^{50} \mathrm{CD} 8+$ cytotoxic T cells and CD4 + T helper (Th) cells are the main $T$ cells that work in the adaptive immune system. CD8 $+\mathrm{T}$ cells are $\mathrm{T}$ cells which are analogous to NK cells and have a role in destroying infected cells but not in recognizing specific antigens. Cytotoxic T cells are activated by kidney-specific autoantigen which can cause kidney disease and inflammation; the active CD8 $+T$ cells can release additional autoantigens resulting in the activation of more CD8 + cytotoxic T cells, which lead to more kidney damage and inflammation. ${ }^{51}$

CD4+ Th cells are activated by inflammatory cytokines and antigens. Th cells consist of Th1, Th2, and Th17. Th1 is marked by the production of inflammatory cytokines such as IFN- $\gamma$, IL-2, lymphotoxin- $\alpha$, and TNF- $\alpha$ and stimulate the activation of macrophage and CD8+ $\mathrm{T}$ cells, leading to tissue damage. On the contrary, Th2 plays an anti-inflammatory role by downregulating Th1 and suppress macrophage activation by producing IL4, IL-5, and IL-10. Th17 cells can induce inflammation in the kidneys by expressing TNF- $\alpha$ and upregulate chemokines which leads to invasion of the kidneys by immune cells. ${ }^{52}$

\section{Renal hemodynamic changes}

The accumulation and activation of leukocytes, monocytes, and macrophages in the kidney lead to hemodynamic changes such as reduced blood flow and glomerular filtration rate (GFR). ${ }^{53}$ Cytokines such as TNF- $\alpha$, TNF- $\beta$, and interleukins can also increase sodium excretion, reduced renal blood flow, and reduced GFR. ${ }^{54}$

\section{Glomerular and tubulointerstitial pathology}

When the kidneys fail to function, complex mechanisms occur thus leading to terminal stage kidney failure. This complex mechanism includes cytokine activity in renal hemodynamic, and glomerulotubular function, which leads to a progressive reduction in GFR.

Mesangial cells plays an important role in glomerular pathology by being a mediator for inflammation. ${ }^{55}$ In a state of immunodeficiency, active mesangial cells release cytokines such as IL-1, RANTES, MCP-1, TGF- $\beta$, and heparin bind to epidermal growth factor (HB-EGF). ${ }^{56}$ Increased cytokines and growth factors result in mesangial cell proliferation, which later secretes extracellular matrix components contributing to tubular sclerosis. ${ }^{57}$ After inflammation, a fibrotic repair will occur. However, in CKD, chronic inflammation causes progressive fibrosis leading to scarring and kidney damage. Tubular cells damaged by lymphocytes and cytokines transform into fibroblasts. These fibroblasts produce extracellular matrix components contributing to over-accumulation of collagen and fibrosis. ${ }^{58}$ 


\section{Diabetes mellitus}

Diabetes mellitus is one of the main causes of ESRD. ${ }^{59}$ Proteinuria and inflammation play a role in tubulointerstitial damage resulting in a progressive decline of GFR. ${ }^{60}$ Immune system activation and chronic inflammation is highly associated with diabetes, which in turn makes diabetes an inflammatory disease. ${ }^{61}$ Inflammation in diabetic kidney disease causes impairment in metabolic transport, renal hemodynamic, and tubular function. Inflammatory mediators such as IL-18 and TNF increased in patients with diabetes and diabetic nephropathy. Studies have shown IL-18 to be a significant marker for diabetic kidney disease progression. ${ }^{62}$

Several studies have found the causative role of acute phase inflammation related to the increase of renal ICAM-1 and MCP-1 in diabetic nephropathy. ${ }^{63}$ ICAM1 tends to interact with $\mathrm{T}$ cells causing migration to the kidneys of diabetics. MCP-1 plays a role in diabetic kidney disease by blocking the MCP-1/CCR2 pathway and inhibits glomerulosclerosis and progression of diabetic nephropathy. ${ }^{64}$ Other inflammatory mediators such as IL-6 increase afterward and thus increases mesangial cells; podocyte IL-6 levels have been linked to the progressivity of diabetic kidney disease $.^{62} \mathrm{IL}-1 \mathrm{lev}-$ els increase in the kidneys of diabetic patients and is associated with the proliferation of glomerular mesangial cells and matrix production. ${ }^{65}$ Despite increasing at the same time-point of disease in the development of diabetic nephropathy both in type 1 and type 2 diabetes, each inflammatory mediator plays a different role in each type of diabetes. ${ }^{62}$

Type 1 diabetes is caused by an idiopathic autoimmune disease causing damage to pancreatic $\beta$-cells, causing hyperglycemia. Hyperglycemia increases the level of NF-B which causes an increase of CAM. ${ }^{66}$ Other inflammatory mediators such as TNF-a, MCP-1, IL-1, IL-6, and MCP-1 also increase under the regulation of NF-B. VEGF levels also increase in type 1 diabetes. ${ }^{67}$ Other factors stimulating kidney damage in type 1 diabetes are AGEs and ROS.

In type 2 diabetes, the difference in the mechanism of kidney damage is the involvement of adipokines, the adipocyte cytokines. Activation of TLR4 in adipocytes is linked to insulin resistance in obese patients with type 2 diabetes, triggering cascades of signals and activates $\mathrm{NF}_{-} \mathrm{B}$ and the release of inflammatory molecules. ${ }^{68}$ TLR 2 and TLR4 also induce the expression of chemokines in tubular epithelial cells. ${ }^{69}$ Obesity and type 2 diabetes lead to the decrease of the anti-inflammatory adiponectin and the increase of the pro-inflammatory leptin and resistin. ${ }^{70}$

\section{Hypertension}

It has been known that immunity contributes to hypertension. According to evidence from the past few years, hypertension has been known to be an inflammatory process that involves both the innate and adaptive immune cells. The immune cells transmigrate and accumulate in the interstitium of the affected tissues and then release cytokines, promoting oxidative stress. Recent data have also elucidated the role of T cells and its various derived cytokines in the development of experimental hypertension. These studies suggest that $\mathrm{T}$ cells that infiltrate the kidneys and perivascular regions are stimulated by angiotensin II, deoxycorticosterone acetate-salt, and excessive catecholamines. There is also an accumulation of monocyte/macrophages in these regions. Cytokines released from macrophages/ monocyte that also accumulate in these areas, including interleukin-17, interferon- $\gamma$, tumor necrosis factor $\alpha$, and interleukin-6, cause renal and vascular disruption, leading to sodium retention and increased systemic vascular resistance. ${ }^{71}$ Immunosuppressing agents such as mycophenolate mofetil (MMF), TNF receptor blocker etanercept, and dexamethasone, prevent the maturation of dendritic cells and $\mathrm{T}$ cell infiltration in double transgenic rats with hypertension which contain human renin and angiotensinogen genes. ${ }^{72} \mathrm{~T}$ cells are more dominant in the role of kidney damage compared to B cells. ${ }^{73}$ Angiotensin II in itself also directly increases the release of renal cytokines, adhesion molecule expression, and production of PAI-1, and also activate NF-KB and reactive oxygen species (ROS) which will increase renal cytokines levels. ${ }^{74}$

A study elucidating the role of T cells in hypertension has shed light on the concept of activated T cells and the release of renal cytokines inducing the increase of blood pressure. A correlation between the level of circulating Th1 cytokines such as TNF and IL-6 and blood pressure has also been found. IL-6 has been known to increase the number of angiotensin I receptors, causing increased sodium absorption and vasoconstriction. ${ }^{75}$ The increased sodium absorption was made possible by the JAK2/STAT3 signal activation in the tubular epithelium caused by IL- 6 . However, IL- 6 was not found to be correlated to decreased renal perfusion in angiotensin II hypertension. ${ }^{76} \mathrm{MCP}-1$ and CCR2 receptor also contribute to renovascular hypertension and kidney damage. ${ }^{77}$

Kidney inflammation associated with obesity was also a contributing factor to hypertension and renal damage; metabolic syndrome will increase inflammation in the kidneys and accelerate CKD. The renal blood flow and glomerular filtration rate in obese individuals tend to increase, leading to increased macrophage infiltration and proinflammatory cytokine levels. ${ }^{78}$ 


\section{Thaha et al}

Cytokine IL-6, TNF- $\alpha$, and MCP-1 increase in hypertensive obese compared to non-obese hypertensive individuals. MCP-1 increases in obese individuals and can affect the proliferation of kidney cells. Reducing the activity of MCP-1 can reduce macrophage infiltration in the kidneys, supporting the theory that MCP-1 contributes to kidney inflammation. ${ }^{79}$

Changes in adipokines such as leptin, adiponectin, and resistin are more profound in hypertensive obese. Leptin can increase reactive oxygen species (ROS) and cause renal endothelial dysfunction. In contrast to leptin, adiponectin has an anti-inflammatory effect and its level decreases in metabolic syndrome, causing further renal damage. Recent studies on resistin also showed its level increases in both genetic and diet-induced obese individuals. The contribution of resistin in renal damage and metabolic syndrome is not fully understood. In general, adipokine alterations associated with obesity will further induce inflammation and damage to the kidneys.

\section{References:}

1. Sun J, Axelsson J, Machowska A., Heimbürger $\mathrm{O}$, Bárány $\mathrm{P}$, Lindholm $\mathrm{B}$, et al. Biomarkers of Cardiovascular Disease and Mortality Risk in Patients with Advanced CKD. Clin J Am Soc Nephrol. 2016 Jul 7;11(7):1163-72.

2. Jankowska M, Cobo G, Lindholm B, Stenvinkel P. Inflammation and Protein-Energy Wasting in the Uremic Milieu. Contrib Nephrol. 2017;191:5871. doi: 10.1159/000479256.

3. Chen L, Deng H, Cui H, Fang J, Zuo Z, Deng J, et al. Inflammatory responses and inflammation-associated diseases in organs. Oncotarget. 2017 Dec 14;9(6):7204-7218.

4. Xu G, Luo K, Liu H, Huang T, Fang X, Tu W. The progress of inflammation and oxidative stress in patients with chronic kidney disease. Ren Fail. 2015 Feb;37(1):45-9.

5. Sayadi M, Pakfetrat M, Sepaskhah M, Roozbeh J, Malekmakan L, et al. Association of high-sensitive C-reactive protein and dialysis adequacy with uremic pruritus. Saudi J Kidney Dis Transpl. 2015 Sep;26(5):890-5.

6. Akchurin, O. and Kaskel, F. (2015). Update on Inflammation in Chronic Kidney Disease. Blood Purif. 2015;39(1-3):84-92.
Indonesian Journal of Kidney and Hypertension

7. Liu Z, Xue L, Liu Z, Huang J, Wen J, Hu J, et al. Tumor Necrosis Factor-Like Weak Inducer of Apoptosis Accelerates the Progression of Renal Fibrosis in Lupus Nephritis by Activating SMAD and p38 MAPK in TGF- $\beta 1$ Signaling Pathway. Mediators Inflamm. 2016;2016:8986451

8 Akdoğan M., Azak A., Denizli N., Huddam B., Koçak G., Gücün M., et al. MCP-1 and soluble TWEAK levels are independently associated with coronary artery disease severity in patients with chronic kidney disease. Ren Fail. 2015;37(8):1297-302.

9. Rhee C., Ahmadi S, Kalantar-Zadeh, K. The dual roles of obesity in chronic kidney disease. Curr Opin Nephrol Hyperten. 2016;25(3):208-16.

10. Lim C., Teo B., Tai E., Lim S., Chan C., Sethi S., et al. Elevated Serum Leptin, Adiponectin and Leptin to Adiponectin Ratio Is Associated with Chronic Kidney Disease in Asian Adults. PLOS ONE. 2015;10(3);p.e0122009.

11. Heidari M, Nasri P, Nasri H. Adiponectin and chronic kidney disease; a review on recent findings . J Nephropharmacol. 2015;4(2):63-8

12. Jiang Y., Zhang J., Yuan Y., Zha X., Xing C., Shen C., et al. Association of Increased Serum Leptin with Ameliorated Anemia and Malnutrition in Stage 5 Chronic Kidney Disease Patients after Parathyroidectomy. Sci Rep. 2016 Jun 16;6:27918

13.Tu J., Cheung W, Mak R. Inflammation and nutrition in children with chronic kidney disease. World J Nephrol. 2016 May 6;5(3):274-82.

14. Caetano, C., Valente, A., Oliveira, T. and Garagarza, C. Body Composition and Mortality Predictors in Hemodialysis Patients. J Ren Nutr. 2016;26(2): 81-6.

15. Sabatino A., Regolisti G., Karupaiah T., Sahathevan S., Sadu Singh B., Khor B., et al.. Protein-energy wasting and nutritional supplementation in patients with end-stage renal disease on hemodialysis. Clinl Nutr. 2017; 36(3): p.663-71.

16. Taraz M, Taraz S, Dashti-Khavidaki S. Association between depression and inflammatory/anti-inflammatory cytokines in chronic kidney disease and endstage renal disease patients: a review of literature. Hemodial Int. 2015 Jan;19(1):11-22. 
Indonesian Journal of Kidney and Hypertension

17. Kraemer, W., Ratamess, N. and Nindl, B. Recovery responses of testosterone, growth hormone, and IGF-1 after resistance exercise. J Appl Physiol. 2017;122(3): 549-58.

18. Cafiero C., Gigante M., Brunetti G., Simone S., Chaoul N., Oranger A., et al. Inflammation induces osteoclast differentiation from peripheral mononuclear cells in chronic kidney disease patients: crosstalk between the immune and bone systems. Nephrol Dial Transplant. 2017;33(1):6575.

19. Munoz Mendoza J., Isakova T., Cai X., Bayes L., Faul C., Scialla J., et al. Inflammation and elevated levels of fibroblast growth factor 23 are independent risk factors for death in chronic kidney disease. Kidney Int, 2017;91(3): 711-9.

20. Chen H., Senda T, Kubo K. The osteocyte plays multiple roles in bone remodeling and mineral homeostasis. Med Mol Morphol. 2015 Jun;48(2):61-8.

21. Oliveira Júnior W., Sabino A., Figueiredo R, Rios, D. Inflammation and poor response to treatment with erythropoietin in chronic kidney disease. J Bras Nefrol. 2015 Apr-Jun;37(2):255-63

22. Nekoui A, Blaise G. Erythropoietin and Nonhematopoietic Effects. AM J Med Sci. 2017;353(1):681.

23. Tucker P., Scanlan A, Dalbo V. Chronic Kidney Disease Influences Multiple Systems: Describing the Relationship between Oxidative Stress, Inflammation, Kidney Damage, and Concomitant Disease. Oxid Med Cell Longev. 2015;2015:806358.

24. Stenvinkel P., Gillespie I., Tunks J., Addison J., Kronenberg F., Drueke T., et al. Inflammation Modifies the Paradoxical Association between Body Mass Index and Mortality in Hemodialysis Patients. J Am Soc Nephrol. 2015;27(5):1479-86.

25. Xuan Y., Wang L., Zhi H., Li X, Wei P. Association Between 3 IL-10 Gene Polymorphisms and Cardiovascular Disease Risk. Medicine. 2016;95(6);e2846.

26. Stinghen AE, Massy ZA, Vlassara H, Striker GE, Boullier A. Uremic Toxicity of Advanced Glycation End Products in CKD. J Am Soc Nephrol. 2015;27(2):354-70
27. Kraut, J. and Madias, N. (2017). Adverse Effects of the Metabolic Acidosis of Chronic Kidney Disease. Advances in Chronic Kidney Disease, 24(5), pp.289-297.

28. Lau, W., Kalantar-Zadeh, K. and Vaziri, N. (2015). The Gut as a Source of Inflammation in Chronic Kidney Disease. Nephron, 130(2), pp.92-98.

29. Stockler-Pinto, M., Saldanha, J., Yi, D., Mafra, D., Fouque, D. and Soulage, C. (2016). The uremic toxin indoxyl sulfate exacerbates reactive oxygen species production and inflammation in 3T3L1 adipose cells. Free Radical Research, 50(3), pp.337-344.

30. Azizieh, F., Alyahya, K. and Raghupathy, R. (2016). Association between levels of vitamin D and inflammatory markers in healthy women. Journal of Inflammation Research, p.51.

31. Kang, Y., Kim, J., Joung, K., Lee, J., You, B., Choi, M., Ryu, M., Ko, Y., Lee, M., Lee, J., Ku, B., Shong, M., Lee, K. and Kim, H. (2016). The Roles of Adipokines, Proinflammatory Cytokines, and Adipose Tissue Macrophages in Obesity-Associated Insulin Resistance in Modest Obesity and Early Metabolic Dysfunction. PLOS ONE, 11(4), p.e0154003.

32. Kuo, I., Wu, P., Lin, H., Niu, S., Huang, J., Hung, C., Chiu, Y. and Chen, H. (2019). The association of adiponectin with metabolic syndrome and clinical outcome in patients with non-diabetic chronic kidney disease. PLOS ONE, 14(7), p.e0220158.

33. Sabatino, A., Regolisti, G., Gandolfini, I., Delsante, M., Fani, F., Gregorini, M. and Fiaccadori, E. (2017). Diet and enteral nutrition in patients with chronic kidney disease not on dialysis: a review focusing on fat, fiber and protein intake. Journal of Nephrology, 30(6), pp.743-754.

34. Cobo, G., Lindholm, B. and Stenvinkel, P. (2018). Chronic inflammation in end-stage renal disease and dialysis. Nephrology Dialysis Transplantation, 33(suppl_3), pp.iii35-iii40.

35. Jing, Z. and Wei-Jie, Y. (2016). Effects of soy protein containing isoflavones in patients with chronic kidney disease: A systematic review and meta-analysis. Clinical Nutrition, 35(1), pp.117124. 
Thaha et al

36. Mikolasevic, I., Žutelija, M., Mavrinac, V. and Orlic, L. (2017). Dyslipidemia in patients with chronic kidney disease: etiology and management. International Journal of Nephrology and Renovascular Disease, Volume 10, pp.35-45.

37. Orlic, L., Mikolasevic, I., Lukenda, V., Anic, K., Jelic, I. and Racki, S. (2014). Nonalcoholic fatty liver disease and the renin-angiotensin system blockers in the patients with chronic kidney disease. Wiener klinische Wochenschrift, 127(9-10), pp.355-362.

38. He, J., Mills, K., Appel, L., Yang, W., Chen, J., Lee, B., Rosas, S., Porter, A., Makos, G., Weir, M., Hamm, L. and Kusek, J. (2015). Urinary Sodium and Potassium Excretion and CKD Progression. Journal of the American Society of Nephrology, 27(4), pp.1202-1212.

39. Machowska, A., Carrero, J., Lindholm, B. and Stenvinkel, P. (2016). Therapeutics targeting persistent inflammation in chronic kidney disease. Translational Research, 167(1), pp.204-213.

40. Liakopoulos, V., Roumeliotis, S., Gorny, X., Dounousi, E. and Mertens, P. (2017). Oxidative Stress in Hemodialysis Patients: A Review of the Literature. Oxidative Medicine and Cellular Longevity, 2017, pp.1-22.

41. Nowak, K., Chonchol, M., Ikizler, T., Farmer-Bailey, H., Salas, N., Chaudhry, R., Wang, W., Smits, G., Tengesdal, I., Dinarello, C. and Hung, A. (2016). IL-1 Inhibition and Vascular Function in CKD. Journal of the American Society of Nephrology, 28(3), pp.971-980.

42. Alves, M., Vilaça, S., Carvalho, M., Fernandes, A., Dusse, L. and Gomes, K. (2015). Resistance of dialyzed patients to erythropoietin. Revista Brasileira de Hematologia e Hemoterapia, 37(3), pp.190-197.

43. Ağbaş, A., Canpolat, N., Çalışkan, S., Yılmaz, A., Ekmekçi, H., Mayes, M., Aitkenhead, H., Schaefer, F., Sever, L. and Shroff, R. (2018). Hemodiafiltration is associated with reduced inflammation, oxidative stress and improved endothelial risk profile compared to high-flux hemodialysis in children. PLOS ONE, 13(6), p.e0198320.

44. Mercadal, L., Franck, J., Metzger, M., Urena Tor-
Indonesian Journal of Kidney and Hypertension

res, P., de Cornelissen, F., Edet, S., Béchade, C., Vigneau, C., Drüeke, T., Jacquelinet, C. and Stengel, B. (2016). Hemodiafiltration Versus Hemodialysis and Survival in Patients With ESRD: The French Renal Epidemiology and Information Network (REIN) Registry. American Journal of Kidney Diseases, 68(2), pp.247-255.

45. Ye, H., Jin, J., Jin, L., Chen, Y., Zhou, Z. and Li, Z. (2016). Chlorogenic Acid Attenuates Lipopolysaccharide-Induced Acute Kidney Injury by Inhibiting TLR4/NF-кB Signal Pathway. Inflammation, 40(2), pp.523-529.

46. O’Neill, L. and Pearce, E. (2015). Immunometabolism governs dendritic cell and macrophage function. The Journal of Experimental Medicine, 213(1), pp.15-23.

47. Cao, Q., Harris, D. and Wang, Y. (2015). Macrophages in Kidney Injury, Inflammation, and Fibrosis. Physiology, 30(3), pp.183-194.

48. Shalapour, S. and Karin, M. (2015). Immunity, inflammation, and cancer: an eternal fight between good and evil. Journal of Clinical Investigation, 125(9), pp.3347-3355.

49. Paloian, N., Leaf, E. and Giachelli, C. (2016). Osteopontin protects against high phosphate-induced nephrocalcinosis and vascular calcification. Kidney International, 89(5), pp.1027-1036.

50. Zewinger, S., Schumann, T., Fliser, D. and Speer, T. (2015). Innate immunity in CKD-associated vascular diseases. Nephrology Dialysis Transplantation, 31(11), pp.1813-1821.

51. Weisheit, C., Engel, D. and Kurts, C. (2015). Dendritic Cells and Macrophages: Sentinels in the Kidney. Clinical Journal of the American Society of Nephrology, 10(10), pp.1841-1851.

52. Heidari, M., Nasri, P. \& Nasri, H., 2015. The role of Th1 and Th17 cells in glomerulonephritis. Journal of Nephropathology, 4(2), pp.63-68.

53. Dellepiane, S., Marengo, M. and Cantaluppi, V. (2016). Detrimental cross-talk between sepsis and acute kidney injury: new pathogenic mechanisms, early biomarkers and targeted therapies. Critical Care, 20(1).

54. Sun, L. and Kanwar, Y. (2015). Relevance of TNF- $\alpha$ 
in the context of other inflammatory cytokines in the progression of diabetic nephropathy. Kidney International, 88(4), pp.662-665.

55. Zhong, S., Zhao, L., Li, Q., Yang, P., Varghese, Z., Moorhead, J., Chen, Y. and Ruan, X. (2015). Inflammatory Stress Exacerbated Mesangial Foam Cell Formation and Renal Injury via Disrupting Cellular Cholesterol Homeostasis. Inflammation, 38(3), pp.959-971.

56. Haller, H., Bertram, A., Nadrowitz, F. and Menne, J. (2016). Monocyte chemoattractant protein-1 and the kidney. Current Opinion in Nephrology and Hypertension, 25(1), pp.42-49.

57. Wu, X., Gao, Y., Cui, F. and Zhang, N. (2016). Exosomes from high glucose-treated glomerular endothelial cells activate mesangial cells to promote renal fibrosis. Biology Open, 5(4), pp.484-491.

58. Gewin, L., Zent, R. and Pozzi, A. (2017). Progression of chronic kidney disease: too much cellular talk causes damage. Kidney International, 91(3), pp.552-560.

59. Ghaderian, S.B. et al., 2015. Diabetes and end-stage renal disease ; a review article on new concepts. , $4(2)$, pp.28-33.

60. Lawson, J., Elliott, J., Wheeler-Jones, C., Syme, H. and Jepson, R. (2015). Renal fibrosis in feline chronic kidney disease: Known mediators and mechanisms of injury. The Veterinary Journal, 203(1), pp.18-26.

61. Grossmann, V., Schmitt, V., Zeller, T., Panova-Noeva, M., Schulz, A., Laubert-Reh, D., Juenger, C., Schnabel, R., Abt, T., Laskowski, R., Wiltink, J., Schulz, E., Blankenberg, S., Lackner, K., Münzel, T. and Wild, P. (2015). Profile of the Immune and Inflammatory Response in Individuals With Prediabetes and Type 2 Diabetes. Diabetes Care, 38(7), pp.1356-1364.

62. Donate-Correa, J., Martín-Núñez, E., Muros-de-Fuentes, M., Mora-Fernández, C. and Navarro-González, J. (2015). Inflammatory Cytokines in Diabetic Nephropathy. Journal of Diabetes Research, 2015, pp.1-9.

63. Sun, L. and Kanwar, Y. (2015). Relevance of TNF- $\alpha$ in the context of other inflammatory cytokines in the progression of diabetic nephropathy. Kidney
International, 88(4), pp.662-665.

64. Wada, J. and Makino, H. (2015). Innate immunity in diabetes and diabetic nephropathy. Nature Reviews Nephrology, 12(1), pp.13-26.

65. Aghadavod, E. et al., 2016. Role of Oxidative Stress and Inflammatory Factors in Diabetic Kidney Disease. , 10(6), pp.337-343.

66. Suryavanshi, S. and Kulkarni, Y. (2017). NF-к $\beta$ : A Potential Target in the Management of Vascular Complications of Diabetes. Frontiers in Pharmacology, 8.

67. Behl, T. and Kotwani, A. (2015). Exploring the various aspects of the pathological role of vascular endothelial growth factor (VEGF) in diabetic retinopathy. Pharmacological Research, 99, pp.137148.

68. Saltiel, A. and Olefsky, J. (2017). Inflammatory mechanisms linking obesity and metabolic disease. Journal of Clinical Investigation, 127(1), pp.1-4.

69. Tang, S., Yiu, W., Lin, M. and Lai, K. (2015). Diabetic Nephropathy and Proximal Tubular Damage. Journal of Renal Nutrition, 25(2), pp.230233.

70. Tong, H., Luu, N., Son, H., Hoan, N., Hung, T., Velavan, T. and Toan, N. (2016). Adiponectin and pro-inflammatory cytokines are modulated in Vietnamese patients with type 2 diabetes mellitus. Journal of Diabetes Investigation, 8(3), pp.295-305.

71. McMaster, W., Kirabo, A., Madhur, M. and Harrison, D. (2015). Inflammation, Immunity, and Hypertensive End-Organ Damage. Circulation Research, 116(6), pp.1022-1033.

72. Moreau, A., Alliot-Licht, B., Cuturi, M. and Blancho, G. (2016). Tolerogenic dendritic cell therapy in organ transplantation. Transplant International, 30(8), pp.754-764.

73. Abais-Battad, J., Rudemiller, N. and Mattson, D. Hypertension and immunity. Curr Opin Nephrol and Hypertens. 2015:24(5); p.470-4.

74. Ratliff, B., Abdulmahdi, W., Pawar, R. and Wolin, M. (2016). Oxidant Mechanisms in Renal Injury and Disease. Antioxid Redox Signal. 2016;25(3):119- 
46.

75. Rincón J, Correia D, Arcaya J, Finol E, Fernández A, Pérez M, et al. Role of Angiotensin II type 1 receptor on renal $\mathrm{NAD}(\mathrm{P}) \mathrm{H}$ oxidase, oxidative stress and inflammation in nitric oxide inhibition induced-hypertension. Life Sci. 2015;124:81-90.

76. Manhiani M., Seth D., Banes-Berceli A., Satou R., Navar L, Brands, M. The role of IL-6 in the physiologic versus hypertensive blood pressure actions of angiotensin II. Physiol Rep. 2015;3(10); e12595.

77. Wang Y, Zhu M, Xu H, Cui L, Liu W, Wang X, Shen S. Wang D. Role of the monocyte chemoattractant protein-1/C-C chemokine receptor 2 signaling pathway in transient receptor potential vanilloid type 1 ablation-induced renal injury in salt-sensitive hypertension. Exp Biol Med. 2015;240(9): 1223-34.

78. Tonneijck L, Muskiet M, Smits M, van Bommel E, Heerspink H, van Raalte D, Joles J. Glomerular Hyperfiltration in Diabetes: Mechanisms, Clinical Significance, and Treatment. J Am Soc Nephrol. 2017;28(4): 1023-39.

79. Haller H., Bertram A., Nadrowitz F, Menne, J. Monocyte chemoattractant protein-1 and the kidney. Curr Opin Nephrol Hypertens. 2016;25(1): 42-9. 